\title{
LA EQUIDAD DE GÉNERO COMO PARTE DE LA RESPONSABILIDAD SOCIAL EN EL MERCADO LABORAL DE LA INDUSTRIA TURÍSTICA DEL ECUADOR
}

\section{RESUMEN}

Actualmente vivimos en un mundo lleno de organizaciones que con su accionar influyen en la vida de las personas y en la sociedad donde se desenvuelven; constituyen un fuerte instrumento de desarrollo económico y social de los países, uno de los ejes que promueve la existencia de las organizaciones corresponde la responsabilidad social vista desde un enfoque ético y moral, donde se promulga la equidad dentro de la sociedad. Esta equidad social se puede visualizar desde una de sus aristas, la equidad de género, y es precisamente des de esta pers pectiva en que se analizará este trabajo, donde todavía se observa desigualdad y discriminación específicamente en el ámbito laboral. La industria turística en el Ecuador no se halla fuera de esta realidad, por ello el objetivo de este estudio es realizar un análisis cuantitativo de la participación de la mujer en el mercado laboral de la industria turística, así como determinar su representación en cargos de decisión y empoderamiento. Para su desarrollo se dividió a la industria turística en actividades turísticas, se determinó los destinos más visitados por turistas nacionales y extranjeros. Los resultados evidencian una desigualdad de género en algunas actividades turísticas, así como una baja representación en cargos de decisión.

Palabras clave: Equidad; Equidad de Género; Igualdad; Turismo; Mercado Laboral Turístico.

\section{EQUIDADE DE GÉNERO COMO PARTE DA RESPONSABILIDADE SOCIAL NO MERCADO DE TRABALHO DA INDÚSTRIA TURÍSTICA DO ECUADOR}

\section{RESUMO}

Atualmente nós vivemos em um mundo cheio de organizações que, com sua ação, influenciam na vida das pessoas e na sociedade onde se desenvolvem; constituem um forte instrumento de desenvolvimento econômico e social dos países, um dos eixos que promove a existência das organizações corresponde à responsabilidade social vista de uma abordagem ética e moral, onde a igualdade é promulgada na sociedade. Esta equidade social pode ser vista de uma das suas bordas, a equidade de gênero, e é precisamente a partir desta perspectiva que este trabalho será analisado, onde ainda há desigualdade e discriminação especificamente na esfera laboral. A indústria do turismo no Equador não está fora dessa realidade, portanto, o objetivo deste estudo é realizar uma análise quantitativa da participação das mulheres no mercado de trabalho da indústria do turis mo, bem como determinar sua representação em pos ições de decisão e capacitação. Para o seu desenvolvimento, a indústria do turismo foi dividida em atividades turísticas, os destinos mais visitados pelos turistas nacionais e estrangeiros foram determinados. Os resultados mostram uma desigualdade de gênero em algumas atividades turísticas, bem como uma baixa representação nas posições de decisão.

Palavras-chave: Equidade; Equidade de Gênero; Igualdade; Turismo; Mercado de Trabalho Turístico. 


\section{THE EQUALITY OF GENDER AS PART OF THE SOCIAL RESPONSIBILITY IN THE TOURIST \\ INDUSTRY LABOUR MARKET OF ECUADOR}

\section{ABSTRACT}

Currently we live in a world with organizations that influence in the people's life and in the society where they operate; the organizations are a strong instrument of economic and social development of the countries, one of them axes that promotes their existence is social the responsibility viewed from an ethical and moral approach, where it is promulgated the equity in the society. This social equality can be analyzed from one of these edges, the equity of gender, and it is precisely from this perspective that I will analyze this work, where we still observed inequality and discrimination specifically in the labor market. The tourist industry in the Ecuador is not out of this reality; the ob jective of this study is performs a quantitative analysis of the woman participation in the tourist industry labor market as determine her representation in decision and empowerment jobs. For its development, this job divided the tourist industry into tour ist activities, destinations more visited by tourists. The results show a gender inequality in some tourist activities, as well as a low representation in positions of decision.

Keywords: Equity; Gender Equality; Equality; Tourism; Tourist Labor Market.

Digna Marcela Viteri Moya ${ }^{1}$ Pablo Guillermo Peñaherrera Mafla ${ }^{2}$ Jenny Lolita Vinueza Luna ${ }^{3}$

\footnotetext{
${ }^{1}$ Maestro en Gerencia Empresarial por la Escuela Politécnica Nacional, Quito - Ecuador. Profesora de la Universidad de las Fuerzas Armadas - ESPE, Ecuador. E-mail: dmviteri@espe.edu.ec

${ }^{2}$ Maestro en Gerencia Empresarial por la Escuela Politécnica Nacional, Quito - Ecuador. Profesor de la Universidad de las Fuerzas Armadas - ESPE, Ecuador. E-mail: pgpeniaherrera@espe.edu.ec

${ }^{3}$ Maestro en Negocios Internacionales por la Universidad Internacional del Ecuador. Profesora de la Universidad de las Fuerzas Armadas - ESPE, Ecuador. E-mail: jlvinueza@espe.edu.ec
} 


\section{INTRODUCCIÓN}

El mayor reto que enfrenta la sociedad actual, es sin duda, el cómo construir sostenibilidad y que a través de su accionar, crear estabilidad y bienestar para su población. Precisamente uno de los pilares que promueve el desarrollo sostenible y del cual se derivan muchas aristas es la responsabilidad social, que de forma general impulsa un comportamiento ético y transparente para con la sociedad y medio ambiente. La Responsabilidad Social se fundamenta en los derechos humanos, siendo uno de los instrumentos que garantiza su consecución la eliminación de todas las formas de discriminación contra la mujer (Norma Internacional ISO 26000, 2010).

Es así como el análisis de la equidad e igualdad de género se fundamenta y se justifica, debido a que su estudio promueve la defensa de los derechos sociales, políticos, económicos tanto de mujeres como de hombres (Naciones Unidas - Fondo de Población de Naciones Unidas, 2006). Bajo esta perspectiva se consideró a Young (2000) una activista en pro de la mujer quien considera que las injusticias institucionalizas que enfrenta la mujer no solo es a nivel nacional sino a nivel global; aborda a la justicia y la política de la diferencia a través de un debate sobre los elementos que considera clave como es el paradigma distributivo y la imparcialidad, poniendo énfasis en la opresión y la desigualdad de género expresados en varias prácticas cotidianas específicamente relacionadas a la distribución del trabajo, la transferencia del poder y toma de decisiones, prácticas que atentan contra la autonomía y el desarrollo pleno de las capacidades de las mujeres; para lo cual propone reorganizar las instituciones, las prácticas de toma de decisiones, modificar la división del trabajo, y tomar medidas similares para el cambio institucional, estructural y cultural.

Meentzen \& Gomariz (2003) sugieren un cambio de paradigma a través de una reorientación estratégica de las relaciones de género que integre necesidades tanto de hombres como de mujeres en el contexto laboral, familiar, doméstico, político; etc.; justificada en la medida en que se genere igualdad de oportunidades de las mujeres en el ámbito laboral y político y donde los hombres accedan a compartir tareas familiares y participen activamente en el proceso de la reorganización democrática de las relaciones entre hombres y mujeres en todos los niveles.

Con lo indicado anteriormente, el objetivo de este estudio es realizar un análisis cuantitativo de la participación de la mujer en el mercado laboral de la industria turística, así como determinar su representación en cargos de decisión y empoderamiento, con la finalidad de efectuar un aporte empírico al estudio de la equidad de género en el mercado laboral turístico. El instrumento que permite analizar a los proveedores dentro del sector turístico es el Catastro Nacional, donde se consolida la información referente a empresas nuevas por actividad, sub-actividad, personal, etc. Este instrumento divide al sector turístico en seis actividades: agencias de viaje; alojamiento; casinos, salas de juegos e hipódromos; comidas y bebidas; recreación, diversión y esparcimiento; y transporte turístico.

Este informe considera las seis actividades turísticas para el análisis, pero limita su comparación a siete provincias, que son consideradas como las más visitadas por turistas nacionales y extranjeros y son éstas provincias quienes aportan con más del $50 \%$ de plazas de empleo dentro del sector turístico. El artículo se encuentra estructurado en cinco apartados: el apartado dos corresponde al desarrollo donde se expone la revisión de literatura de elementos importantes relacionados a la equidad de género; el apartado tres vincula las hipótesis seleccionadas de la teoría; el apartado cuarto desarrolla la metodología; el apartado quinto presenta el análisis de los resultados empíricos y la discusión; el apartado sexto se compone de las conclusiones; y finalmente se presenta la lista de referencias.

\section{MARCO TEÓRICO}

Definir el término turismo es un reto, pues su actividad produce un efecto multiplicador que involucra varios sectores; según la Organización Mundial del Turismo, el turismo es considerado como: un fenómeno social, cultural y económico relacionado con el movimiento de las personas a lugares que se encuentran fuera de su lugar de residencia habitual por motivos personales o de negocios/profesionales. Estas personas se denominan visitantes (que pueden ser turistas o excursionistas; residentes o no residentes) y el turismo tiene que ver con sus actividades, de las cuales algunas implican un gasto turístico (Organización Mundial del Turismo , 2016).

Es decir en el turismo intervienen algunos actores, el primero corresponde al turista y como lo menciona Panosso (2007) el turismo envuelve seres que son turistas y seres que no son turistas, seres que podrán ser turistas y seres que ya fueron turistas; para que sea denominado turista conlleva en si una experiencia, por ello el turismo se considera un fenómeno de experiencias vividas de maneras y formas diferentes (pág. 391)

Los otros actores, o mejor dicho elementos, constituyen la planta turística, los atractivos turísticos, los prestadores de servicio, etc.; pero todos ellos no existirían sin que el ser humano decida darles un valor a través de su experiencia, su importancia radica en que el turismo se ha vuelto una fuente de ingresos para quienes forman parte de esta compleja estructura, 
haciendo que los países busquen nuevas estrategias para diferenciar la imagen de su país, invertir en campañas turísticas, buscarnuevos nichos de mercados y aperturar nuevos destinos para de esta forma atraer a los turistas; el Ecuador en este sentido ha sabido promocionar su riqueza al mundo combinando varios aspectos mencionados dentro del Sumak Kawsay.

El Sumak Kawsay, desde la perspectiva de la cosmovisión andina, recoge una visión del mundo centrado en el ser humano (Ministerio de Educación Ecuador), corresponde a un principio transversal constitucional cuyo fin es el garantizar la sostenibilidad y el buen vivir de su población, este principio de sostenibilidad pretende hacer posible no solo la permanencia de la diversidad cultural y ambiental, sino también la felicidad individual y colectiva (Secretaria del Buen Vivir , 2016); que trasladado a las empresas, ha obligado a las mismas a buscar e implementar estrategias que les as egure su supervivencia a futuro, $y$ es así como una de estas herramientas denominada Responsabilidad Social, aparece para construir desde la perspectiva empresarial lazos que integren sus objetivos con los objetivos de la sociedad.
La definición de Responsabilidad Social ha sido estudiada desde algunos enfoques, unos, relacionados principalmente con la ética y con la integración con la sociedad; Garriga y Melé (2004) mencionan que el término responsabilidad social puede agruparse en cuatro teorías fundamentales: teorías instrumentales, teorías políticas, teorías integradores y teorías éticas. Las dos primeras teorías expresan que el objetivo principal de la empresa es la creación de la riqueza, entendiéndose como la responsabilidad de la empresa la de maximizar utilidades para los accionistas y la creación de ventaja competitiva; así como el poder social que puede gestionar la empresa dentro de la sociedad; mientras que las teorías integradoras y éticas están relacionadas principalmente con la integración de las demandas sociales y los valores éticos que deben considerar las empresas y organizaciones para lograr un beneficio común entre las partes interesadas, en ellas se vincula el principio de equidad, que promueve el beneficio mutuo, la justicia, y la cooperación (Garriga \& Melé, 2004)

Figura 1 - Teorías que agrupan a las definiciones de Responsabilidad Social

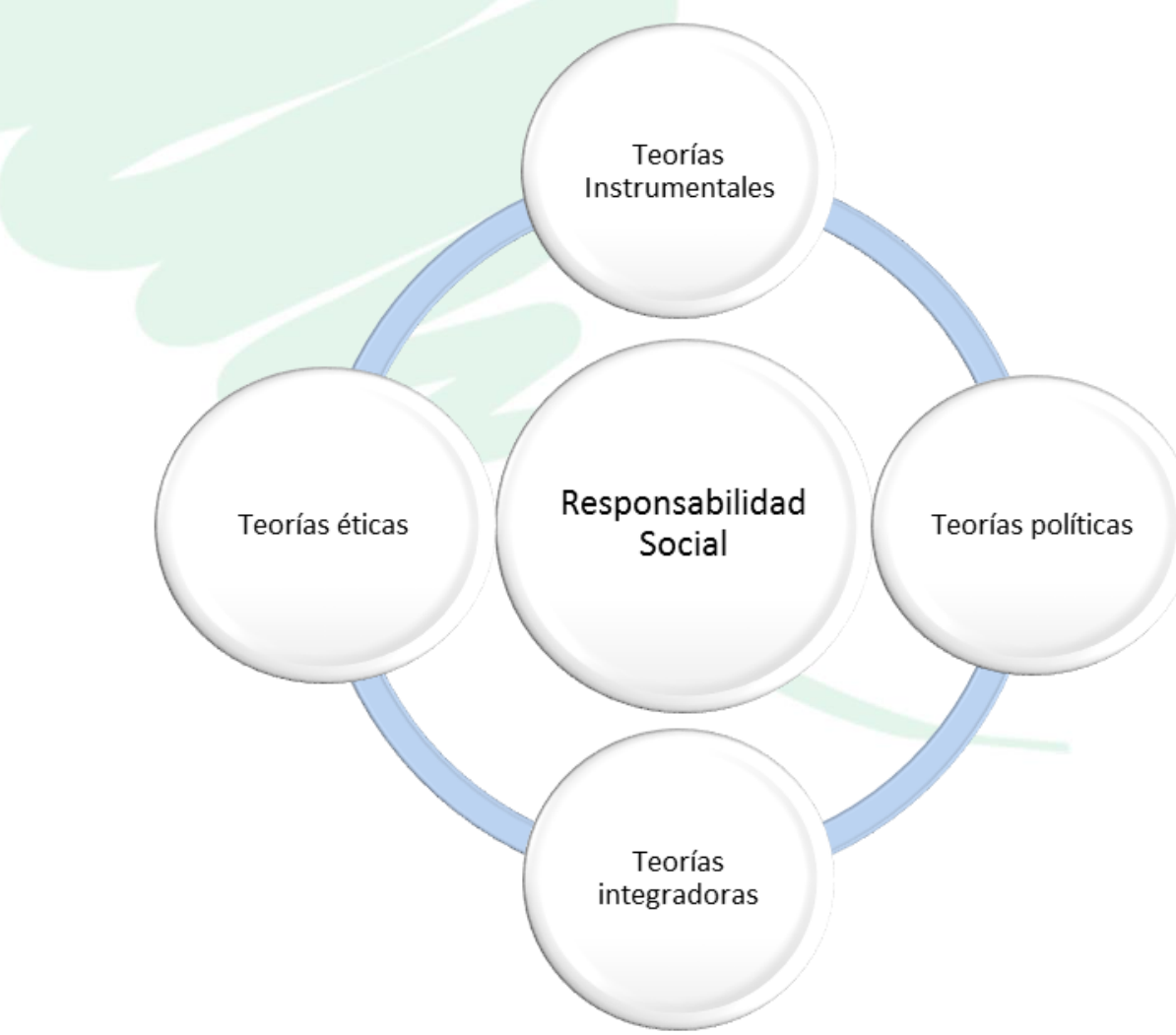

Fuente: Adaptación (Garriga \& Melé, 2004)

Estas últimas definiciones están sustentadas en la Declaración de los Derechos Humanos cuyo objetivo principal es que todo ser humano debe tener derechos y libertades sin distinción, este principio constituye uno de los pilares del desarrollo sostenible. (Consejo de Derechos Humanos de la Organización de las Naciones
Unidas, 1948); entre los instrumentos internacionales que tratan sobre los derechos humanos se encuentran siete, que constituyen la base de las normas internacionales de los derechos humanos universales, y que se mencionan a continuación. 
Figura 2 - Instrumentos internacionales en materia de Derechos Humanos

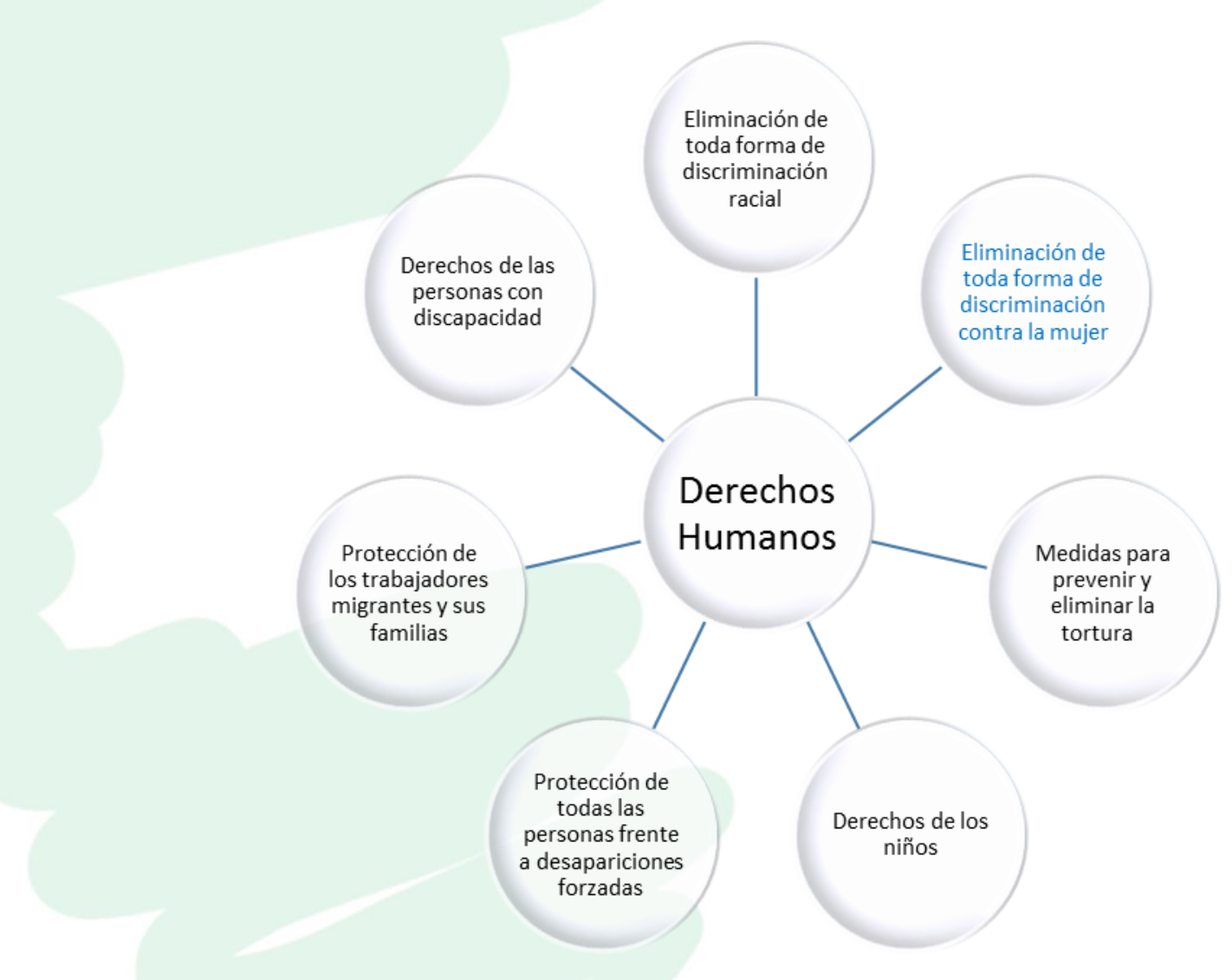

Fuente: Adaptación (Norma Internacional ISO 26000, 2010)

De igual manera, la Norma ISO 26000 - Guía de Responsabilidad Social, considera dentro de sus principios el respeto a los derechos humanos y como principio adicional la promoción de la igualdad de género y empoderamiento de la mujer, este elemento es considerado también como uno de los objetivos del milenio, siendo un compromiso asumido por la ONU en su agenda 2030 para el desarrollo sostenible (Naciones Unidas, 2015).

Ahora bien, existen muchas acepciones que tratan de explicar el término género y su diferencia con el término sexo; Stoller, expresó que el género era un término netamente cultural, mientras que el sexo era una expresión natural (Palomino, 2010); De Barbieri (1993) expresa que analizar al género como un producto de las relaciones sociales del sexo, podría causar desigualdad entre hombres y mujeres. Banchs (2010) menciona que el género constituye el resultado de una construcción social del ser humano; entonces podríamos plantear que el género visto desde una perspectiva social y cultural permite visualizar al ser humano tanto de forma individual como colectiva, permitiendo comprender de esta manera sus relaciones sociales, de poder y su identidad dentro de la sociedad.

Las relaciones de género tienden a resaltar la desigualdad de género en relación directa a la mujer como un problema frecuente que se observa en todos los países, evidenciándose las situaciones de discriminación femenina, que han conllevado a establecer políticas y acciones pertinentes a la erradicación de este problema. Young ${ }^{4}$ (2000), una feminista reconocida, realiza un amplio debate sobre la justicia social, muestra su preocupación por la situación de la mujer a nivel global, expresando que la opresión de la mujer como injusticia social está determinada por cinco elementos: la explotación, la marginación, la carencia de poder, el imperialismo cultural androcéntrico, y la violencia. La explotación

\footnotetext{
4 Young, I.M., título original de la obra Justice and the Politics of Difference, Princeton University Press, Princeton, 1990; se encuentra citada la traducción en español de Silvina Álvarez, La Justicia y la Política de la Diferencia, Ediciones Cátedra, Universidad de Valencia, Instituto de la Mujer, Madrid, 2000
} 
relacionada directamente con el trabajo, sea este remunerado o no, donde la mujer transfiere energía y poder a otro grupo, entendiéndose como otro grupo, a los hombres; hace referencia a que las mujeres por lo general se relacionan con trabajos que suministran placer, bienestar, prestación de cuidados a otras personas, entre otras actividades, sin que el trabajo sea remunerado adecuadamente; resaltando de esta manera la existencia institucionalizada en la sociedad de trabajos considerados aptos para mujeres y otros aptos para hombres.

La marginación está relacionada con aquellos grupos que se encuentran al margen de una sociedad hegemónica, consintiendo privaciones, discriminaciones, y dependencias, precisamente uno de estos grupos constituyen las mujeres. La carencia del poder está relacionada directamente con la explotación y menciona que este elemento se percibe en aquellas personas sin formación o trabajadoras (es) sin profesionalización que no poseen autonomía para tomar sus propias decisiones. El imperialismo cultural androcéntrico, según la autora, está dado cuando los rasgos de la cultura dominante impiden visualizar la perspectiva particular de las mujeres. Finalmente la violencia está enlazada directamente con la violencia de género, que dependiendo de la cultura, puede ser tolerada y legitima.

Esta llamada injusticia social causa en las relaciones de género, relaciones desiguales de poder, y se traducen en pobreza, diferencia en la distribución de la riqueza y de espacios (Mesa de Género de la Cooperación Española en Ecuador, 2011).

Meentzen \& Gomariz (2003) proponen una reorientación estratégica en las relaciones de género expresando que se genere igualdad de oportunidades de las mujeres en el ámbito laboral y político y que los hombres accedan a compartir tareas familiares y participen activamente en el proceso de la reorganización democrática de las relaciones entre hombres y mujeres en todos los niveles.

Young $^{5}$ (1993), sugiere una distinción entre necesidades prácticas e intereses estratégicos de género; las primeras relacionadas con los roles sexuales asignados a la mujer y que son similares entre las culturas y están vinculados con la alimentación, cuidado y educación de los hijos, entre otros; mientras que los intereses estratégicos de género cuestionan la situación de la mujer en la sociedad y por consiguiente la desigualdad generada principalmente por el control masculino del trabajo de las mujeres, el acceso limitado de las mujeres a los recursos económicos y sociales y

\footnotetext{
5 Resumido y editado por Jennifer L. Newton para su publicación. El texto en el cual se basa se escribió en 1989 y se publicó como el capítulo 9, "Planning from a gender perspective : Making a world of difference", en el libro de Kate Young, Planning Development with Women: Mak ing a World o f D ifference. London: Macmillan, 1993. Traducido por Adriana Espinosa y Magdalena León.
}

al poder político, siendo el resultado final una distribución desigual de los recursos entre los géneros.

Como se mencionó en párrafos anteriores, la equidad de género promueve un desarrollo sostenible en la sociedad, entendiéndose como desarrollo sostenible el propuesto en el Informe Brundtland (1989), el cual expresa que "el desarrollo sostenible es aquel que satisface las necesidades de la generación presente sin comprometer la capacidad de las generaciones futuras para satisfacer sus propias necesidades", frente a esta premisa las organizaciones y empresas deberían crear herramientas que les permita tener un enfoque de desarrollo humano sostenible y un enfoque de género que vayan de la mano con la agenda internacional del desarrollo. (Plan de Acción de Género en Desarrollo de la Cooperación Española en Ecuador 2011-2014, 2011)

Entre las herramientas que contribuyen a generar oportunidades laborales a la mujer son sin duda el empoderamiento y la transversalidad que por un lado permite a la mujer percibirse como capaz y merecedora de ocupar un espacio en la toma de decisiones y por otro lado se incluye la prioridad de igualdad de género en cada estrategia y sector no solo a nivel país sino a nivel empresarial (Cano \& Arroyabe, 2014). Esta estrategia de empoderamiento fue propuesto inicialmente por grupos activistas feministas cuyas demandas estaban relacionadas a cambiar radicalmente los procesos y las estructuras que hacían que la mujer tenga una posición subordinada como género; esta posición exigía una postura más colectiva que individual y consideraba la reconstrucción de una autoimagen y autoconfianza positiva de la mujer, el desarrollo de la habilidad para pensar críticamente, la construcción de una relación más estrecha como grupo y la promoción para que la mujer tenga el control de su propia vida y pueda tomar decisiones (Young K. , 1993), (Kabeer, 1994)

\section{HIPÓTES IS}

Para determinar las hipótesis o más bien proposiciones, se consideró una de las premisas propuestas por Young (2000) en la que pone en manifiesto su criterio de oposición frente a la división de trabajo y la explotación pues afirma que existe aún la creencia de que ciertos trabajos están diseñados y son socialmente aceptados como femeninos o masculinos.

Por lo tanto se consideró determinar si la actividad turística, agencias de viaje, es aquella donde se refleja mayor participación de la mujer, y si la actividad turística, transporte turístico, refleja menor participación de la mujer. 


\section{METODOLOGÍA}

La investigación exploratoria es defendida por Bryman (2012) mencionando que es una estrategia de investigación en las ciencias sociales que permite visualizar un fenómeno dentro del contexto de la vida real. Expresa que para comprender los fenómenos que ocurren en el ámbito social se requiere de teorías, por lo tanto éstas tienen influencia tanto en lo que se investiga como en los hallazgos; de ahí que hace referencia sobre la naturaleza de la relación que existe entre la teoría y la investigación, mostrando que cuando la teoría guía la investigación se sugiere al investigador realizar un acercamiento deductivo, es decir la temática a investigar está influenciada por posiciones teóricas disponibles. Aunque este tipo de investigación no permita establecer una generalización estadística, sin embargo permite construir proposiciones a partir de un conjunto particular de resultados.

Figura 3 - Proceso de la teoría deductiva, relación natural entre la teoría y la investigación social

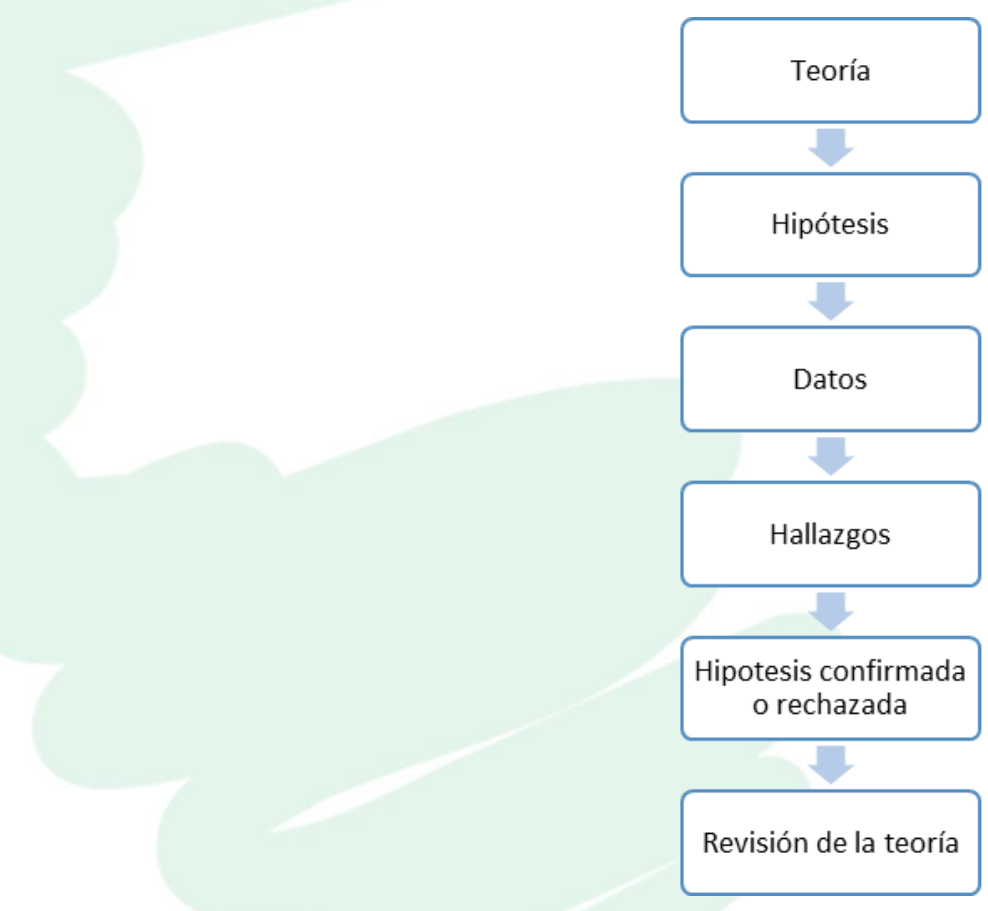

Fuente: Adaptación (Social Research Methods, 2012, pág. 24)

En esta investigación, la metodología utilizada fue de tipo documental, se analizó datos secundarios proporcionados por el Ministerio de Turismo del Ecuador, este método es sugerido por la facilidad de realizar análisis longitudinales, siendo posible trazar tendencias y conexiones a través del tiempo.

Se utilizó tablas dinámicas, conocidas también como tablas pivot en Excel, debido a la posibilidad de organización de la información en columnas y filas, así como la comparación de información en cuestión de segundos.

Para el análisis se utilizó cuatro etapas:

Etapa 1: Se obtuvo datos de fuentes secundarias fiables, proporcionadas por el ente rector de la actividad turística en Ecuador, esta información corresponde al Catastro Nacional de Turismo; se consideró para el estudio los datos desde el año 2010 hasta el año 2016.
Etapa 2: Se escogió los principales destinos turísticos, de acuerdo a la información del Ministerio de Turismo, Migración y Ministerio del Ambiente.

Etapa 3: La información se la trató tomando en cuenta los siguientes datos:

$\checkmark$ Actividad y sub-actividad turística

$\checkmark \quad$ Número de trabajadores hombres

$\checkmark \quad$ Número de trabajadores mujeres

$\checkmark \quad$ Período de análisis desde el año 2010 - 2016

Etapa 4: Se escogió al azar 47 empresas turísticas grandes, considerando el número de colaboradores que posee cada empresa, con la finalidad de visualizar la presencia de la mujer en cargos directivos (gerencia general, subgerencia, presidencia, vicepresidencia), esta información se realizó considerando el año 2015. 
La Equidad de Género como Parte de la Responsabilidad Social en el Mercado Laboral de la Industria Turística del Ecuador

En la tabla 1, se observa el número de empresas turísticas divididas por actividad turística que se consolidó desde el año 2010 al 2016, así como el porcentaje de crecimiento en número de empresas y por actividad durante el período de análisis.

Tabla 1 - Número de empresas turísticas divididas por actividad turística período 2010 - 2016

\begin{tabular}{|c|c|c|c|c|c|c|c|c|}
\hline $\begin{array}{l}\text { Actividad } \\
\text { Turística }\end{array}$ & $\begin{array}{c}\text { Año } \\
2010\end{array}$ & $\begin{array}{l}\text { Año } \\
2011\end{array}$ & $\begin{array}{c}\text { Año } \\
2012\end{array}$ & $\begin{array}{c}\text { Año } \\
2013\end{array}$ & $\begin{array}{c}\text { Año } \\
2014\end{array}$ & $\begin{array}{c}\text { Año } \\
2015\end{array}$ & $\begin{array}{c}\text { Año } \\
2016\end{array}$ & $\begin{array}{c}\text { Porcentaje de } \\
\text { crecimiento } \\
\text { período 2010-2015 }\end{array}$ \\
\hline $\begin{array}{l}\text { Agencias de } \\
\text { viajes }\end{array}$ & 1.148 & 1.272 & 1.457 & 1.545 & 1.661 & 1.866 & 1.943 & $69 \%$ \\
\hline Alojamiento & 3.099 & 3.493 & 4.264 & 4.601 & 5.207 & 5.493 & 5.064 & $63 \%$ \\
\hline $\begin{array}{c}\text { Casinos, Salas } \\
\text { de juego e } \\
\text { Hipódromos } \\
\end{array}$ & & & 1 & 1 & 1 & 1 & 1 & $0 \%$ \\
\hline $\begin{array}{c}\text { Comidas } y \\
\text { bebidas }\end{array}$ & 9.012 & 10.166 & 12.704 & 13.255 & 15.359 & 16.775 & 16.693 & $85 \%$ \\
\hline $\begin{array}{c}\text { Recreación, } \\
\text { diversión, } \\
\text { esparcimiento }\end{array}$ & 543 & 599 & 805 & 919 & 1039 & 1190 & 1.223 & $125 \%$ \\
\hline $\begin{array}{c}\text { Transporte } \\
\text { turístico }\end{array}$ & 230 & 273 & 358 & 376 & 401 & 446 & 453 & $97 \%$ \\
\hline Total general & 14.032 & 15.803 & 19.589 & 20.697 & 23.668 & 25.771 & 25.377 & $81 \%$ \\
\hline
\end{tabular}

Fuente: Elaboración propia en base a (Catastro Nacional Turístico - Ministerio de Turismo)

En la tabla 2 se muestran las provincias más visitadas, en estos lugares se encuentran varios atractivos que los turistas visitan, y que mencionaremos a continuación.

Tabla 2 - Principales provincias visitadas por turistas nacionales y extranjeros durante el año 2015

\begin{tabular}{|c|c|}
\hline Turistas & Destinos turísticos en Provincias \\
\hline Turistas Nacionales & $\begin{array}{c}\text { Guayas, Pichincha, Tungurahua, Manabí, } \\
\text { Esmeraldas }\end{array}$ \\
\hline Turistas Extranjeros & $\begin{array}{c}\text { Pichincha, Guayas, Galápagos, Imbabura y } \\
\text { Cotopaxi }\end{array}$ \\
\hline
\end{tabular}

Fuente: Elaboración propia en base a (Ministerio de Turismo, Ministerio del Ambiente, Migración)

Se consideró importante resaltar los atractivos turísticos que son visitados por turistas nacionales y extranjeros en las provincias mencionadas anteriormente. 
La Equidad de Género como Parte de la Responsabilidad Social en el Mercado Laboral de la Industria Turística del Ecuador

Tabla 3 - Principales atractivos turísticos visitados

\begin{tabular}{|c|c|}
\hline $\begin{array}{l}\text { Principales provincias } \\
\text { visitadas }\end{array}$ & Principales atractivos turísticos visitados \\
\hline Guayas & Guayaquil / Isla Puná / Isla Santay, etc. \\
\hline Pichincha & Quito / Mitad del Mundo / Mindo / Machachi, etc. \\
\hline Tungurahua & $\begin{array}{c}\text { Ambato / Baños / Quisapincha / Parque Nacional LLanganates / } \\
\text { Parque Nacional Sangay/ Cevallos / Píllaro, etc. }\end{array}$ \\
\hline Manabí & $\begin{array}{c}\text { Playas de: Canoa / San Vicente / Manta /Puerto Cayo / Los Frailes / } \\
\text { Parque Nacional Machalilla / Isla de la Plata / Puerto López / } \\
\text { Ayampe, etc. }\end{array}$ \\
\hline Esmeraldas & Playas de: Mompiche / Atacames / Tonsupa / Same /Sua, etc. \\
\hline Imbabura & $\begin{array}{c}\text { Otavalo / Cotacachi / Ibarra / Lago de San Pablo / Cascada de } \\
\text { Peguche / Valle del Chota, etc. }\end{array}$ \\
\hline Galápagos & $\begin{array}{c}\text { Conjunto de is las a las cuales se puede acceder en embarcaciones } \\
\text { como cruceros o barcos más pequeños, estas is las son visitadas por } \\
\text { la flora y fauna única en el mundo. }\end{array}$ \\
\hline
\end{tabular}

Fuente: Elaboración propia en base a (Ministerio de Turismo)

En la tabla 4 se visualiza el número de empresas analizadas considerando el sector turístico al que pertenecen, este análisis permitió verificar la presencia de la mujer en la toma de decisiones de las empresas, esto es ocupando cargos directivos.

Tabla 4 - Empresas analizadas por sector turístico y provincia, para observar la presencia de la mujer en cargos de dirección

\begin{tabular}{|c|c|c|c|c|c|}
\hline Provincia & $\begin{array}{c}\text { Agencias de } \\
\text { Viaje }\end{array}$ & Alojamiento & $\begin{array}{c}\text { Comidas y } \\
\text { Bebidas }\end{array}$ & $\begin{array}{c}\text { Recreación, diversión y } \\
\text { esparcimiento }\end{array}$ & $\begin{array}{c}\text { Total de } \\
\text { empresas }\end{array}$ \\
\hline Guayas & 1 & 2 & 1 & 1 & 5 \\
\hline Pichincha & 4 & 3 & 1 & 0 & 8 \\
\hline Tungurahua & 1 & 1 & 1 & 0 & 3 \\
\hline Manabí & 4 & 3 & 1 & 0 & 8 \\
\hline Esmeraldas & 2 & 2 & 1 & 0 & 5 \\
\hline Imbabura & 2 & 2 & 1 & 0 & 8 \\
\hline Galápagos & 2 & 4 & 2 & 0 & 5 \\
\hline Cotopaxi & 2 & 2 & 1 & 1 & 47 \\
\hline Total & 18 & 19 & 9 & & 5 \\
\hline
\end{tabular}

Fuente: Elaboración propia en base a (Superintendencia de Compañías del Ecuador 2016) 


\section{ANÁLISIS Y DISCUSIÓN DE RESULTADOS}

Pese a los cambios efectuados en la constitución, en donde se incorpora y garantiza la igualdad entre hombres y mujeres, y la eliminación de cualquier forma de discriminación contra la mujer; se observa que en el sector laboral de la industria turística aún existe rasgos de discriminación en algunas actividades turísticas consideradas como netamente de mujeres o de hombres, debido al rol que se genera en cada una de ellas.

Se observa que en actividades de oficina, se encuentra mayoritariamente representada por la mujer (actividades de agencias de viajes) teniendo una participación del $52 \%$ frente al $48 \%$ de participación del hombre en este tipo de actividades; mientras que en aquellas actividades cuyos roles requieren esfuerzo físico como las actividades de recreación, diversión, esparcimiento; así como transporte turístico, es más representativo la presencia del hombre, siendo la participación de la mujer en promedio del $38 \%$ en estas actividades. Estos datos en cierto modo permiten visualizar la realidad de un sector durante siete años desde el año 2010 hasta el año 2016, y con ello confirmar las proposiciones propuestas inicialmente que aún sigue existiendo actividades turísticas catalogadas para hombres y para mujeres considerando el sexo más que el género.

Esta investigación permitió un conocimiento preliminar de lo que ocurre en el sector laboral turístico en relación a la equidad de género; sin embargo todavía quedan varias preguntas por responder, entre ellas el obtener una visión desde la perspectiva del hombre, de la propia mujer; determinar adicionalmente algunas variables como su nivel de escolaridad frente a los cargos de dirección y decisión; el sueldo frente al trabajo, la división de trabajo doméstico tanto de hombres como de mujeres, cuidado y educación de hijos, el tiempo libre, etc; y complementar el estudio considerando un enfoque estadístico.

\subsection{Empleo por género de acuerdo a la actividad turística}

De acuerdo al análisis de los datos expuesto en la tabla 5, se evidencia que más del $50 \%$ de trabajos en promedio generados en el sector turístico son ocupados por hombres; hay actividades turísticas donde pese a existir una diferencia en la participación de hombres y mujeres en el mercado laboral, no es elevada y se observa más bien una complementariedad.

Tabla 5 - Porcentaje de empleos por género de acuerdo a actividad turística período 2010 - 2016

\begin{tabular}{|c|c|c|c|c|c|}
\hline Actividad Turística & $\begin{array}{c}\text { Promedio } \\
\text { hombres } \\
\text { Período } \\
2010 \text {-2016 }\end{array}$ & $\begin{array}{c}\text { Promedio } \\
\text { Mujeres } \\
\text { Período } \\
2010 \text {-2016 }\end{array}$ & $\begin{array}{c}\text { Total } \\
\text { Promedio hombres } \\
\text { y mujeres } \\
\text { Período } \\
2010 \text {-2016 }\end{array}$ & $\begin{array}{c}\text { \% Hombres } \\
\text { Período } \\
2010-2016\end{array}$ & $\begin{array}{l}\text { \% Mujeres } \\
\text { Período } \\
2010-2016\end{array}$ \\
\hline Agencias de Viajes & 3.420 & 3.691 & 7.111 & $48 \%$ & $52 \%$ \\
\hline Alojamiento & 15.544 & 13.850 & 29.394 & $53 \%$ & $47 \%$ \\
\hline $\begin{array}{c}\text { Casinos, Salas de juego } \\
\text { e Hipódromos }\end{array}$ & 16 & 0 & 16 & $100 \%$ & $0 \%$ \\
\hline Comidas y Bebidas & 32.987 & 30.448 & 63.435 & $52 \%$ & $48 \%$ \\
\hline $\begin{array}{l}\text { Recreación, diversión, } \\
\text { esparcimiento }\end{array}$ & 3.362 & 1.791 & 5.153 & $65 \%$ & $35 \%$ \\
\hline Transporte turístico & 1.784 & 793 & 2.577 & $69 \%$ & $31 \%$ \\
\hline $\begin{array}{c}\text { Total promedio } \\
\text { empleos por actividad }\end{array}$ & 57.114 & 50.572 & 107.686 & $53 \%$ & $47 \%$ \\
\hline
\end{tabular}

Fuente: Elaboración propia en base a (Ministerio de Turismo - Catastro Nacional Turístico anual) 
5.2 Empleos por género considerando las provincias más visitadas por turistas

Como se observa en la tabla 6 las provincias que aportan con más empleo en el sector turístico del Ecuador son Guayas y Pichincha con el $76 \%$ de la participación total de las provincias más visitadas; en ellas se evidencia que el hombre tiene una mayor participación aunque la diferencia en realidad es mínima; sin embargo se denota también una discriminación laboral por el género, en provincias donde predomina el trabajo físico como es el caso de la provincia de Galápagos, donde la participación de la mujer en la actividad laboral corresponde al 38\% del promedio total de empleos generados en la provincia; Galápagos es una de las provincias más visitadas por extranjeros, seguramente porque se ofrece a los turistas la realización de actividades marinas muy apetecidas como viajes en cruceros, viajes en barcos, actividades de snorkel, kayak, buceo, surfing, entre otras; así como también actividades en tierra como caminatas largas, avistamiento de aves, etc. Todas estas actividades son aceptadas socialmente como actividades para los hombres.

Por otro lado se evidencia la existencia de provincias que presentan una participación elevada de la mujer en el sector laboral turístico como es el caso de las provincias de Cotopaxi, Imbabura, Tungurahua y Esmeraldas, probablemente esta situación se dé por la presencia de rasgos de una cultura propia, donde existen comunidades rurales y campesinas que han desarrollado como alternativa económica de progreso el turismo comunitario y responsable; ofertan principalmente servicios relacionados a valorar su patrimonio cultural y natural y donde la mujer definitivamente tiene alta participación; en la mayoría de casos son actividades socialmente aceptadas para mujeres.

Tabla 6 - Porcentaje de empleos por género considerando las provincias más visitadas por turistas nacionales y extranjeros período 2010 - 2016

\begin{tabular}{|c|c|c|c|c|c|c|}
\hline Provincia & $\begin{array}{c}\text { Promedio } \\
\text { Hombres } \\
\text { Período } \\
2010 \text {-2016 } \\
\end{array}$ & $\begin{array}{l}\text { Promedio } \\
\text { Mujeres } \\
\text { Período } \\
\mathbf{2 0 1 0}-\mathbf{2 0 1 6} \\
\end{array}$ & $\begin{array}{c}\text { Total promedio } \\
\text { Período } \\
2010 \text {-2016 }\end{array}$ & $\begin{array}{l}\text { \% Hombres } \\
\text { Período } \\
2010 \text {-2016 }\end{array}$ & $\begin{array}{l}\text { \% Mujeres } \\
\text { Período } \\
2010 \text {-2016 }\end{array}$ & $\begin{array}{c}\text { \% de aportación } \\
\text { de empleo al } \\
\text { mercado laboral } \\
\text { turístico }\end{array}$ \\
\hline Esmeraldas & 1.117 & 1.279 & 2.397 & $47 \%$ & $53 \%$ & $3 \%$ \\
\hline Galápagos & 1.456 & 893 & 2.349 & $62 \%$ & $38 \%$ & $3 \%$ \\
\hline Imbabura & 1.616 & 1.854 & 3.470 & $47 \%$ & $53 \%$ & $4 \%$ \\
\hline Manabí & 3.008 & 2.701 & 5.709 & $53 \%$ & $47 \%$ & $7 \%$ \\
\hline Pichincha & 17.315 & 14.678 & 31.993 & $54 \%$ & $46 \%$ & $38 \%$ \\
\hline Tungurahua & 2.195 & 2.521 & 4.716 & $47 \%$ & $53 \%$ & $6 \%$ \\
\hline $\begin{array}{c}\text { Total promedio } \\
\text { empleos por } \\
\text { provincia } \\
\end{array}$ & 45.330 & 38.302 & 83.632 & $54 \%$ & $46 \%$ & $100 \%$ \\
\hline
\end{tabular}

Fuente: Elaboración propia en base a (Ministerio de Turismo - Catastro Nacional Turístico anual)

\subsection{Cargos directivos de acuerdo al género}

Conforme se menciona en la teoría, una estrategia de desarrollo de la mujer en lo referente al género es sin duda el empoderamiento, tomando en consideración su derecho a ser coparticipe del desarrollo sostenible del país; bajo esta perspectiva se analizó la situación actual que tiene la mujer en puestos de decisión y de dirección en el sector laboral turístico; en los datos analizados que se muestran en la figura 3, se observa que un $69 \%$ de cargos directivos son ocupados por hombres, frente al $31 \%$ de cargos directivos ocupados por mujeres, es decir se evidencia un predomino del varón en cargos directivos.

Esta situación seguramente se da porque el hombre aún es considerado como principal actor de desarrollo económico y su participación es socialmente aceptada y relativamente mejor valorada para funciones directivas y de toma de decisiones. Por otro lado considerando que una proporción importante de empresas tienen una alta composición familiar, refleja el comportamiento patriarcal de esta estructura organizacional y cultural. 
La Equidad de Género como Parte de la Responsabilidad Social en el Mercado Laboral de la Industria Turística del Ecuador

Tabla 7 - Cargos directivos de acuerdo al género

\begin{tabular}{|c|c|c|c|c|c|}
\hline Cargos directivos & Hombre & $\begin{array}{c}\text { Porcentaje cargos } \\
\text { directivos hombres }\end{array}$ & Mujer & $\begin{array}{c}\text { Porcentaje cargos } \\
\text { directivos mujeres }\end{array}$ & $\begin{array}{c}\text { Total } \\
\text { empresas }\end{array}$ \\
\hline $\begin{array}{c}\text { Presidente / } \\
\text { vicepresidente }\end{array}$ & 34 & $72 \%$ & 13 & $28 \%$ & 47 \\
\hline Gerente / subgerente & 31 & $66 \%$ & 16 & $34 \%$ \\
\hline Total & $\mathbf{6 5}$ & $\mathbf{6 9 \%}$ & $\mathbf{2 9}$ & $\mathbf{3 1 \%}$ & $\mathbf{4 7}$ \\
\hline
\end{tabular}

Fuente: Elaboración propia en base a (Superintendencia de Compañías del Ecuador)

Figura 3 - Cargos directivos de acuerdo al género en la industria turística

\section{Cargos directivos de acuerdo al género en la industria turística}

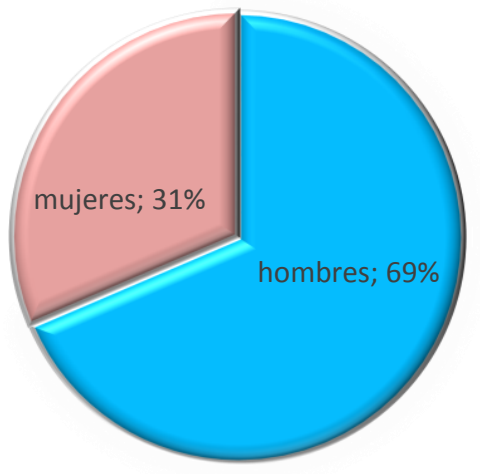

Fuente: Elaboración propia en base a (Superintendencia de Compañías del Ecuador)

Ahora bien, si se analiza con mayor detalle los cargos directivos por provincia con mayor afluencia de turistas nacionales y extranjeros, se observa que existe un mayor predominio del hombre en cargos de dirección en las provincias de Guayas, Esmeraldas, Galápagos, Pichincha, Tungurahua, cuyas cifras son alarmantes, por ejemplo en la provincia del Guayas el 90\% de los cargos directivos del sector turístico corresponde al género masculino. Nuevamente se observa un patrón cultural de aceptación y valoración de características relacionadas al poder y toma de decisiones que posee el hombre en el ámbito empresarial, es decir varias de estas organizaciones continúan siendo estructuras no solo jerárquicas de clase sino de sesgo de género y que varios aspectos como el acceso al capital y otros recursos siguen siendo no negociables para la mujer. 
Figura 4 - Cargos directivos de acuerdo al género por provincia

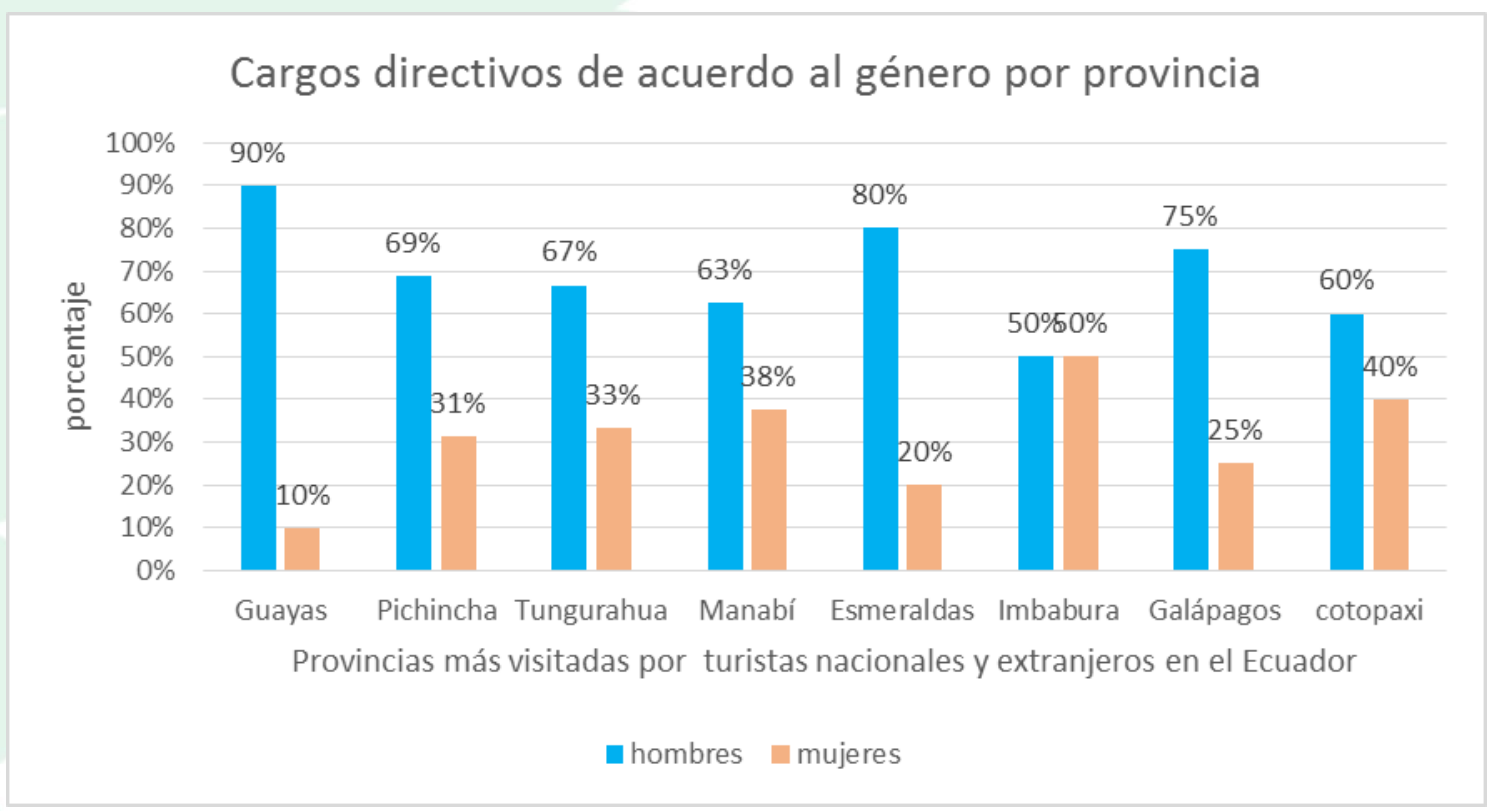

Fuente: Elaboración propia en base a (Superintendencia de Compañías del Ecuador)

\section{CONCLUSIONES}

Este estudio de caso permite visualizar que pese a los esfuerzos realizados por los gobiernos, organizaciones, etc.; todavía existe desigualdad de género en el trabajo dentro del mercado laboral turístico; continúa la creencia que existe roles que están supeditados al género ya sea hombre o mujer (Young I. , 2000) (Young K. , 1993).

Esto es evidente en las actividades de transporte turístico, de recreación, diversión y esparcimiento; y de casinos, salas de juego e hipódromos donde la diferencia es abismal; mientras que en las actividades de agencias de viaje, alojamiento, comidas y bebidas es más equilibrado.

También se observa que en provincias donde predomina las comunidades existe un "matriarcado", estas provincias son Cotopaxi, Esmeraldas, Imbabura y Tungurahua.

En provincias como Galápagos la diferencia de género en el mercado laboral es grande, seguramente porque en esta provincia existen actividades turísticas que ameritan un mayor esfuerzo físico y existe la creencia que estas actividades son para "hombres" no para "mujeres".

Algo similar ocurre al analizar la distribución de los cargos de decisión por género, donde se observa mayoritariamente la presencia del varón, dejando de lado a la mujer.

Este ha sido un primer acercamiento a la equidad de género en el mercado laboral turístico del Ecuador, sin embargo, se recomienda para próximas investigaciones realizar un diagnóstico para analizar la gestión administrativa del hombre y de la mujer y su incidencia en el mercado, así como integrar el resto de provincias al estudio; también se sugiere considerar una comparación entre los cargos administrativos vs. los cargos de dirección.

\section{REFERENCIAS}

Banchs, M. (2010). http://webs.uvigo.es/pmayobre. Recuperado el 10 de Julio de 2016, de http://pmayobre.webs.uvigo.es/textos/maria_banchs /representaciones_sociales_del_genero_en_discurso _politico.pdf

Brundtland, G. (1989). Global change and our common future. Science and policy for sustainable development Taylor and Francis Group, 16 - 43.

Bryman, A. (2012). Social Research Methods. New York: Oxford University Press.

Cano, T., \& Arroyabe, O. (2014). Procesos de empoderamiento de mujeres, subjetivación y transformaciones en las relaciones de poder. Revista virtual Universidad Católica del Norte .

Consejo de Derechos Humanos de la Organización de las Naciones Unidas. (10 de Diciembre de 1948). Consejo de Derechos Humanos de la Organización de las Naciones Unidas. Recuperado el 2016 de Julio de 9, de www.ohchr.org: http://www.ohchr.org/EN/UDHR/Documents/UDH R_Translations/spn.pdf 
De Barbieri, T. (1993). Sobre la categoría de género, una introducción teórico - metodológica . Instituto de investigaciones de la UNAM, debates en Sociología No. 18.

Garriga, E., \& Melé, D. (2004). Corporate Social Responsability. Journal of Business Ethics , 51-71.

Género y educación, a. a. (2015). Trimiño, Celina. Revista historia educación latinoamericana, 55-72.

Kabeer, N. (1994). Reversed realities: Gender Hierarchies in development thought. Londres: Verso.

Meentzen, A., \& Gomariz, E. (2003). Democracia de género, una propuesta inclusiva. El Salvador: Fundacion Heinrich Boll.

Mesa de Género de la Cooperación Española en Ecuador. (2011). Plan de Acción de Género en Desarrollo de la Cooperación Española en Ecuador 2011-2014. Obtenido de http://www.upec.edu.ec/dmdocuments/DESCARG ASUPEC/PlanGeneroenDes arrollo2011-2014.pdf

Ministerio de Turismo. (Agosto de 2016). Ministerio de Turismo. Obtenido de http://www.turismo.gob.ec/catastro-deestablecimientos-turisticos/

Naciones Unidas - Fondo de Población de Naciones Unidas. (Diciembre de 2006). Recuperado el 10 de Julio de 2016, de http://www.entremundos.org/databases/Herramient as $\% 20 \mathrm{de} \% 20$ trabajo $\% 20$ en $\% 20$ genero $\% 20$ UNFPA .pdf

Naciones Unidas. (Septiembre de 1995). www.un.org. Obtenido de http://www.un.org/womenwatch/daw/beijing/pdf/B DPfA\%20S.pdf

Naciones Unidas. (25 de Septiembre de 2015). http://www.un.org/. Obtenido de Transformar nuestro mundo: Agenda 2030 para el desarrollo sostenible; resolución aprobada por la Asamblea
General el 25 septiembre 2015: http://www.un.org/es/comun/docs/?symbol=A/RES $/ 70 / 1$

Norma Internacional ISO 26000. (01 de 11 de 2010). www.fundahrse.org. Recuperado el 9 de Julio de 2016, de http://fundahrse.org

Organización Mundial del Turismo . (12 de febrero de 2016). Organización Mundial del Turismo. Obtenido de http://media.unwto.org/es/content/entender-elturismo-glos ario-basico

Palomino, M. (Octubre de 2010). Suplemento Memorias $V \quad$ Encuentro. Obtenido de https://academia.unad.edu.co/images/investigacion/ hemeroteca/revistainvestigaciones/Volumen9numer o2 2010/36.\%20PERCEPCIONES,\% 20IMAGINA RIOS.pdf

Panosso, A. (2007). Filosofía del Turismo, una propuesta epistemológica. Estudios y Perspectivas en Turismo, 389-402.

Programa de las Naciones Unidas para el desarrollo. (2014). Obtenido de http://hdr.undp.org/sites/default/files/2015_human_ development_report_overview_-_es.pdf

Secretaria del Buen Vivir . (15 de Agosto de 2016). Secretaria del Buen Vivir. Obtenido de http://www.secretariabuenvivir.gob.ec/el-buenvivir-en-la-constitucion-del-ecuador/

Superintendencia de Compañias del Ecuador. (Agosto de 2016). Superintendencia de Compañias del Ecuador. Obtenido de http://www.supercias.gob.ec/portalinformacion/con sulta/

Young, I. (2000). La Justicia y la Política de la diferencia. Madrid - España: Ediciones Cátedra (Grupo Anaya S.A).

Young, K. (1993). Planning development with women: Making a world of difference. London: Macmillan. 